\title{
Decoupling of crystalline and conformational degrees of freedom in lipid monolayers
}

Ipsen, John Hjorth; Mouritsen, Ole G.; Zuckermann, Martin J.

Published in:

Journal of Chemical Physics

Link to article, DOI:

10.1063/1.457090

Publication date:

1989

Document Version

Publisher's PDF, also known as Version of record

Link back to DTU Orbit

Citation (APA):

Ipsen, J. H., Mouritsen, O. G., \& Zuckermann, M. J. (1989). Decoupling of crystalline and conformational degrees of freedom in lipid monolayers. Journal of Chemical Physics, 91(3), 1855-1865.

https://doi.org/10.1063/1.457090

\section{General rights}

Copyright and moral rights for the publications made accessible in the public portal are retained by the authors and/or other copyright owners and it is a condition of accessing publications that users recognise and abide by the legal requirements associated with these rights.

- Users may download and print one copy of any publication from the public portal for the purpose of private study or research.

- You may not further distribute the material or use it for any profit-making activity or commercial gain

- You may freely distribute the URL identifying the publication in the public portal

If you believe that this document breaches copyright please contact us providing details, and we will remove access to the work immediately and investigate your claim. 


\title{
Decoupling of crystalline and conformational degrees of freedom in lipid monolayers
}

\author{
John Hįt lpsen and Ole G. Mouritsen \\ Department of Siructural Properties of Marerials, The Technical Universizy of Denmark. Building 307. \\ DK-2800 Lyngby, Denmark
}

Martin \&. Zuckermann

Department of Physics, RcGill University, Montreals Quebec H3A 278, Canada

(Received 16 September 1988; accepted 14 April 1989)

\begin{abstract}
A theoretical study is performed on a microscopic interaction model which describes the transitions between liquid and solid phases of lipid monolayers spread on air/water interfaces. The model accounts for condensation in terms of acyl-chain conformational degrees of freedom as well as in terms of variables which describe the onientations of crystalline domains in the solid. The phase behavior of the model as a function of temperature and lateral pressure is explored using mean-field theory and computer-simulation techniques. Attention is paid to the particular interplay between the two types of condensation processes and effects on the phase behavior due to decoupling of crystalline and conformational order parameters. In the case of decoupling, the model predicts that the high-pressure solid-conformationally ordered phase is separated from the low-pressure liquid-conformationally disordered phase by a liquidconformationally ordered phase. This prediction is consistent with synchrotron $x$-ray experiments which show that the chain-ordering transition and the crystallization process need not take place at the same lateral pressure. A characterization is provided of the nonequilibrium effects and pattern-formation processes observed along the isotherms in the phase diagram spanned by lateral pressure and area. A description is given of the kinetics of the nonequilibrium phase transitions and the concomitant heterogeneous microstructure of the monolayer. This leads to an explanation of the peculiarities of the experimentally observed isotherms of lipid monolayer phase behavior. It is pointed ont that cholesterol, which promotes lipid-chain conformational order, has a unique capacity of acting as a ${ }^{6}$ crystal breaker in the solid monolayer phases and therefore provides a molecular mechanism for decoupling crystalline and conformational order in lipid monolayers containing cholesterol. The phase diagram of mixed cholesterol-lipid monolayers is derived and discussed in relation to monolayer experiments.
\end{abstract}

\section{INTRODUCTIOK}

Lipid monolayers spread on an air-water interface and the cooperative phenomena they display have been the subject of a vast number of experimental investigations since the pioneering work of Adam. ${ }^{1}$ The predominant experimental technique was for a long time the measurement of lateral pressure (IV)-surface area ( $A$ ) isotherms using a Langmuir trough. ${ }^{2}$ A detailed analysis of the isotherms indicates that lipid monolayers exhibit a varied phase behavior. ${ }^{3}$ There are in fact several related phase transitions and the most signifcant of these are (i) the transition of the monolayer from a liquid-expanded (LE) phase to a liquid condensed (LC) phase at $\Pi_{m}$ and (ii) a solid-solid transition between the LC phase and a highly condensed solid phase at $\Pi_{c}$ which appears as a sharp shoulder in the monolayer isotherm at high lateral pressure. The LC/LE phase transition has been regarded as an analog to the main gel-to-fiuid (liquid crystalIine) phase transition of lipid bilayers ${ }^{4}$ with the following phase correspondence. The bilayer fluid phase and the monolayer $\mathrm{L} E$ phase have both been considered as liquid phases in which the acyl chains of the lipid molecules are in a conformationally disordered state having several gauche bonds.
The bilayer gel phase and the monolayer $L C$ phase are both characterized in terms of straight acyl chains in a conformationally ordered state, but the positional order of these phases is not equivalent in that the acyl chains of a bilayer in the gel phase lie on a two-dimensional crystalline lattice, ${ }^{5,6}$ whereas recent experiments show that the monolayer LC phase has at best long-range bond-orientational order and short-range positional order. ${ }^{7-9}$ There is now experimental evidence to show that a monolayer phase with long-range positional order and conformationally ordered chains exists at high lateral pressures. ${ }^{7}$ This implies that the degrees of freedom associated with the positional (crystalline) order and the conformational states of the chains are not coupled in some pure lipid monolayers whereas they are coupled in pure lipid bilayers.

The experimental methods which give a detailed picture of monolayer structure are of three types. The first method involves electron microscopy on frozen Langmuir-Blodgett films which are deposited on a solid substate ${ }^{10,11}$ and the second method is fuorescence microscopy on in situ monolayers doped with dye molecules. ${ }^{12-15}$ The third method is used by Kjaer et al. ${ }^{7}$ who made the first successful study of in situ monolayers of dimyristoyl phosphatidic acid (DMPA) 
on an air-water interface using the new technique of synchrotron $x$-ray diffaction. This study led to the identification of the solid-solid transition as a first-order phase transition to a crystalline monolayer phase at high lateral pressures. A subsequent study by Helm et al." combined this technique with fluorescence microscopy and data from lateral pressure-area isotherms to show that the LC phase has bondorientational order over tens of micrometers whereas the positional correlations have a range of the order of nanometers only. A reflectivity study by the same authors ${ }^{16}$ led to the conclusion that the acyl chains in the crystalline phase of dipalmitoyl phosphatidylcholine (DPPC) is close packed but that the chains are tilted with respect to the interfacial nomal. Furthermore, optical second-harmonic generation has shown the molecules to reorient upon compression ${ }^{17}$ and more recently vibrational spectroscopy indicated that the molecules in the LC phase are extended and oriented normal to the interface. ${ }^{18}$

The purpose of this article is to present and analyze a theoretical model which considers both the crystalline and conformational degrees of freedom. Previous theoretical analysis of the LC/LE transition concentrated on the conformational degrees of freedom oniy. ${ }^{19-24}$ The most recent of these theories is an examination of monolayer phase equil:bria by the present authors ${ }^{24}$ in which the phase equilibria of the monolayer are described in terms of a ten-state conformational model due to $\mathrm{Pink}^{25}$ and the monolayer properties were obtained by means of a numerical simulation using a Monte Carlo importance-sampling technique. The results showed that the Pink model led to an understanding of the nonkorizontal nature of the monolayer isotherms at the $\mathrm{LC} /$ LE transition in terms of nonequiliorium effects involving strong lateral density fuctuations. ${ }^{24}$ This is consistent with the recent equiliorium experiments of Pallas and Pethica ${ }^{26}$ which led to horizontal isotherms indicating that the $\mathrm{LC} /$ LE transition is a well-defined first-order phase transition. Even though the calculated isotherms gave a good fit to experimental results, the theory was not able to give an understanding of the positional degrees of freedom in monolayers. ${ }^{24}$ In this paper we present an extension of the lattice model of Ref. 24 with the following features: (i) the conformational states of the acyl chains are described by Pink's tenstate model, (ii) the positional degrees of freedom are represented by crystal orientational variables by a high- $Q$-state Potts model, and (iii) the effects of intrinsic impurities, e.g., cholesterol, on both the positional and conformational degrees of freedom are explicitly included. This model was previously applied to the related probiem of crystallisation and acyl-chain ordering in lipid bilayers. ${ }^{6}$ Furthermore, a condensed version of some of the work reported here has already appeared in letter form. ${ }^{27}$

The strategy adopted in Ref. 24 involved the use of computer simulation techniques to study the effect of density fuctuations related to changes in conformation of the acyl chains on the monolayer isotherms. In this paper the same simulation techniques are used to derive the statistical mechanical and thermodynamic properties of the model described by (i)-(iii) above which incorporates both confor mational and positional degrees of freedom. These methods of calculation, which are in principle exact, are supplemented by calculations based on the mean-field approximation. The model on which we have based our study is a lattice model neglecting true translational variables and it cannot account for two-dimensional hexatic phases. ${ }^{28}$ The strength of the lattice-model approach is that it permits a detailed study of the phase behavior, even in the presence of foreign molecules such as cholesterol. A couple of computer-simulation studies based on molecular dynamics methods have recently been reported. ${ }^{29,30}$ These studies which incorporate chain conformational as well as true positional variables give detailed information about conformational changes and packing properties but are not able to treat sufficiently large systems to approach the phase transitions.

The paper is arranged as follows. In Sec. II we present the microscopic interaction model for lipid monolayer phase transitions in terms of two Hamiltonians, one for the conformational states of the acyl chains and the other for the $Q$ state Potts model which we use to model the crystalline degrees of freedom. Section III contains a detailed description of the mean-field approximation and presents the mean-field phase diagram for this model. We continue in Sec. IV with a brief description of the numerical methods used to simulate the model. We then present the phase diagram, the isotherms, and the physical properties of the monolayers resuiting from numerical data obtained by these simulations. Particular attention is paid to nonequilibrium phenomena and pattern-formation processes such as crystallization and grain growth in the neighborhood of the high-pressure tran sition. Section $V$ gives a comparison of the theoretical results with experimental data for pure lipid monolayers. Section VI contains a description of the effect of cholesterol on the phase behavior of lipid monolayers and a modification of the Hamiltoniar of the model for pure lipid monolayers so as to inchude cholesterol. A discussion of the manner in which the mean-field phase diagram for lipid-cholesterol monolayers can be calculated is presented in this section. The paper is concluded in Sec. VII.

\section{औ. MICROSCOPIC MODEL}

The calculations presented below are based on the tenstate conformational model of $P$ ink, ${ }^{25}$ which describes chain melting in lipid monolayers and bilayers, combined with a multistare Potts model which is used to treat the rranslational degrees of freedom in an approximate manner. The ten-state model has been described in great detail in previous publications ${ }^{19,25}$ and requires at mos a brief introduction. This model is a two-dimensional triangular lattice model which accounts for the intrachain conformational energy, the rotational isomerism of the chains, and the van der Waals interaction between chains. The ten chain conformations allowed for in the model include an all-trans ground state, eight gel-like excited states and a highly excited state of low conformational order characteristic of the LE phase. Each conformational siate is characterized by a cross-sectional area per molecule $A_{n}$, an internal conformational energy $E_{n}$, which is related to the number of gauche defects, and a degeneracy $D_{n}$, which can be regarded as a single- 
chain density of states. The ten-state model on its own describes a phase transition from a conformationally ordered to a conformationally disordered phase which is driven by the difference between the high conformational entropy of the excited state and the considerably lower conformational entropies of the gellike states.

The multistate Potts model used here is a modification of the standard high-Q-state Potts model which has had some success in the description of graing growth in polycrys. talline aggregates. ${ }^{31}$ The standard Potts model accounts for the energy of the grain boundaries of a metastable distribution of crystalline domains, each of which is characterized by a Potts state. In the modified Potts model used below, each lattice site represents an acyl chain of the lipid molecule and carries two independent sets of states, i.e., $Q$ Potts states and ten configurational states. The Botts variables describe the orientation of the crystalline domain with which the chain on the lattice site is associated. Since the crystalline domains can be formed in a very large number of orientations, $Q$ has to be a large number. We have chosen $Q=30$ which is known to be a good representation of the large- $Q$ limit. ${ }^{27,31}$ The domain-boundary energy is modeled by allowing neighboring acyl chains to interact with an energy $\mathscr{J}_{p}>0$ if they are in different Pots states and if at the same time each of the interacting chains is in one of the nine gel-like states. Otherwise the interaction is zero. This is reasonable since the tenth conformational state is representative of the $L E$ phase which is fluid and which therefore cannot have a gramular nature. The modified Potts model therefore in a very approximate manner accounts for the translational degrees of freeden of the solid phase. It cannot account for more involved effects such as for example bond-orientational order.

The ten-state model and the $Q$-state Potts model each govern their own first-order phase transition in terms of their respective variables. In the combined model, these two transitions can be either coupled or uncoupled.

The total Hamiltonian of the monolayer can now be written as follows:

$$
\mathscr{H}_{\text {tot }}=\mathscr{H}_{\text {conf }}+\mathscr{H}_{P} \text {, }
$$

where $\mathscr{H}_{\text {com }}$ is the Hamiltonian of the ter-state Pink model for the conformational states of the chains and can be written in the following form

$$
\mathscr{H}_{\mathrm{conf}}=\mathscr{H}_{C}+\mathscr{H}_{C C}+\Pi \sum_{i} \sum_{n=1}^{10} A_{n} \mathscr{L}_{i n}
$$

In $\mathrm{Eq}$. (2) II is the lateral external pressure exerted on the monolayer, $i$ is a site index for the lattice, and $\mathcal{L}_{i n}$ is a state occupation variabie which is unity when the the chain is in the $n$th conformational state and zero otherwise. $\mathscr{K}_{C}$ is the Hamiltonian for the intrachain energies given by

$$
\mathscr{H}_{c}=\sum_{i} \sum_{n=i}^{10} E_{n} Z_{i n} \text {. }
$$

$\mathscr{H}_{c} c$ is the Hamiltonian for the effective var der Waals interaction between neighboring chains and is written as follows:

$$
\mathscr{H}_{C C}=-\frac{\bar{J}_{0}}{2} \sum_{\langle i, j} \sum_{m, n=1}^{10} I_{m} I_{n} \mathscr{S}_{i m} \mathscr{L}_{j n}
$$

where $i$ and $j$ are nearest-neighbor indices, $l_{0}$ is the coupling constant of the van der Waals interaction between acyl chains in the all-trans state and $I_{m}$ depends on $A_{m}$. A detailed expression for $I_{m}$ can be found in $R$ ef. 25 . The values of all parameters pertaining to $\mathscr{F}_{\text {con }}$ except $J_{0}$ are those which were previously determined from applications to the chain melting transition of lipid bilayers. ${ }^{32}$

$\mathscr{H}_{P}$ is the Hamilonian for the modified $Q$-state Potts model and is written as follows:

$$
\mathscr{H}_{P}=\frac{\mathscr{J}_{p}}{2} \sum_{\langle, i)} \sum_{m, n=1}^{Q} \sum_{p, q=1}^{Q}\left(1-\mathcal{S}_{p q}\right) \mathscr{L}_{i p}^{P} \mathscr{L}_{j q}^{P} \mathscr{L}_{i m} \mathscr{L}_{j n}
$$

$J_{P}$ is the coupling constant of the Potts interaction and $\mathscr{L}_{i p}^{P}$ is an occupation variable which is unity when the chain on the $i$ th lattice site is associated with the pth Potts state.

\section{HE. MEAN-FIELD CALCULATIONS}

\section{A. Mean-fleld theory}

In this section we examine the model of the previous section in the mean-field approximation. The resulting mean-field phase diagram and isotherms are described in Sec. III $\mathrm{B}$.

The Gibbs free energy can in general be written in the form

$$
G(T, \Pi)=\langle\mathscr{T}\rangle-T S
$$

where $\langle\mathscr{H}\rangle$ is the expression for the internal energy as the thermal average of the Hamiltonian and $S$ is the entropy of the system which is generally written

$$
S=-k_{B} T\langle\ln \rho\rangle .
$$

$\rho$ is the equilibrium distribution function. In the mean-field approximation the sites of the lattice are treated as statistically independent, and $\rho$ can therefore be written

$$
\rho=\prod_{i}^{N} \rho_{i},
$$

where $\rho_{i}$ is the distribution function at site $i$, and $N$ is the number of lattice sites. Further, since the ramiltonian for the pure monoiayer is translationally invariant, $\rho_{i}$ rakes on the same value $\bar{\rho}$ at every site $i$. From Eqs. (7) and (8), the entropy now becomes

$$
S / N=-k_{B} T\langle\operatorname{in} \bar{\rho}\rangle .
$$

The internal energy, $\langle\mathscr{f}$ tot $\rangle$, for pure lipid monolayers can be written as follows in the mean-field approximation

$$
\begin{aligned}
& \left\langle\mathscr{H}_{\text {tot }}\right\rangle / N=\sum_{n=1}^{10}\left(E_{n}+\Pi A_{n}\right)\left\langle\mathscr{C}_{n}\right\rangle \\
& -J_{0} \frac{z}{2} \sum_{n, n^{\prime} \ldots 1}^{10} I_{n} I_{n^{\prime}}\left\langle\mathscr{L}_{n}\right\rangle\left\langle\mathscr{Z}_{n^{\prime}}\right\rangle \\
& +\xi_{p} \frac{z}{2} \sum_{n, q^{2}-1}^{g} \sum_{q, q^{2}=1}^{q}\left(1-\delta_{q, q^{\prime}}\right) \\
& \times\left\langle\mathscr{L}_{q}^{P} \mathscr{L}_{n}\right\rangle\left\langle\mathscr{L}_{q}^{P} \mathscr{L}_{n^{\prime}}\right\rangle .
\end{aligned}
$$

$z$ is the coordination number for the lattice, $z=6$. The meanfield equilibrium distribution function is obtained from minimizing the Gibbs free cnergy of Eq. (6) with respect to $\bar{\rho}$. 
The following equation for $\bar{p}$ is then obtained from Eqs. $(6)$, (9), and (10)

$$
\bar{\rho}=\frac{\exp \left[-h /\left(k_{B} T\right)\right]}{\operatorname{Tr}\left\{\exp \left[-h /\left(k_{B} T\right)\right]\right\}}
$$

where

$$
\bar{h}=\frac{\delta\left(\left\langle\mathscr{H}_{\text {tot }}\right\rangle / N\right)}{\delta \bar{\rho}}
$$

Use of Eq. (11) leads to a set of self-consistent nonlinear equations involving mean values of occupation variables and their combinations, e.g.,

$$
\left\langle\mathscr{L}_{n} \mathscr{L}_{q}^{P}\right\rangle=\frac{D_{n} \exp \left[-\bar{E}_{n q} /\left(k_{B} T\right)\right]}{\mathbb{Z}}
$$

for $n=1, \ldots, 9, q=1, \ldots, Q$, and

$$
\left\langle\mathscr{Z}_{10}\right\rangle=\frac{D_{10} Q \exp \left[-\bar{E}_{10} /\left(k_{B} T\right)\right]}{Z}
$$

$Z$ is the single-site partition function which is given by

$$
\begin{aligned}
Z & =\sum_{n=1}^{9} D_{n} \sum_{q} \exp \left[-\bar{E}_{n q} /\left(k_{B} T\right)\right] \\
& +Q D_{10} \exp \left[\bar{E}_{10} /\left(k_{B} T\right)\right] .
\end{aligned}
$$

where

$$
\begin{aligned}
& \bar{E}_{n q}=E_{n}+\amalg A_{n}-z J_{0} I_{n} \sum_{n^{\prime}=1}^{10} I_{n^{\prime}}\left\langle\mathscr{L}_{n^{\prime}}\right\rangle \\
& \quad+z J_{P} \sum_{n^{\prime}=1}^{g}\left(\left\langle\mathscr{L}_{n^{\prime}}\right\rangle-\left\langle\mathscr{L}_{n^{\prime}} \mathscr{L}_{q}^{P}\right\rangle\right), \\
& n=1, \ldots, 9, q=1, \ldots, Q, \text { and } \\
& \bar{E}_{10}=E_{10}+\Pi A_{10}-z J_{0} I_{10} \sum_{n^{\prime}=1}^{10} I_{n^{\prime}}\left\langle\mathscr{L}_{n^{\prime}}\right\rangle .
\end{aligned}
$$

Equations (13)-(17) are solved numerically yielding values for the occupation variables.

Since the mean-field theory only yields equilibrium states of the system it cannot be used to describe the noneguilibrium properties of lipid monolayers, particularly their granular nature as observed in the high-pressure phases. This is the task of the numerical simulations which are described in Sec. IV.

\section{B. Mean-field phase diagrams and isotherms}

The mean-fieid equations of Sec. III A are used to obtain the phase diagram at a fixed temperature in terms of lateral pressure and strength: of the Potts interaction $J_{p}$. The results presented in this section are obtained for a set of model parameters for the conformational part of the Hamiltonian, Eq. (2), which is pertinent to phospholipids with saturated chains of 16 carbon atoms, e.g., DPPC. In Fig. 1 are shown the phase diagrams for three selected temperatures. Figure 1 shows that the phase diagram has a region where the two phase transitions of the model are coupled as well as a region where they are decoupled. The decoupling gives rise to a triple point for a particular value $J_{P}=J_{i}^{*}$. For $J_{P}>J_{P}^{*}$ there are two phases separated by one phase boundary. The phase at low lateral pressures is characterised by Potts disorder and conformationally disordered chains, i.e, acyl chains

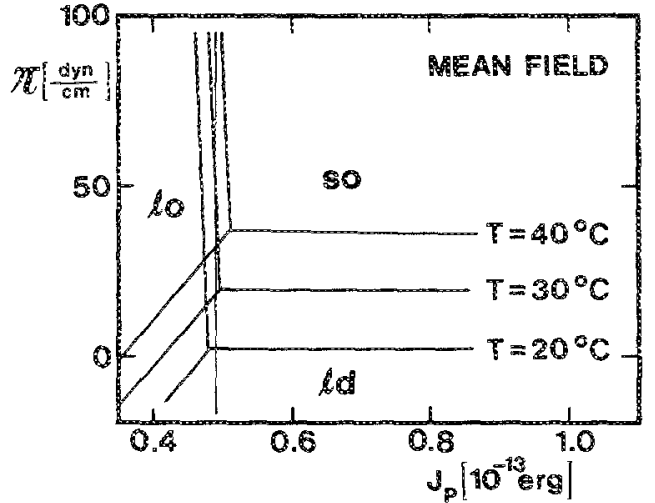

FIG. 1. Mean-field phase diagrams, viz. lateral pressure II vs Potts coupling constan $f_{p}$, for three different temperatures. All phase lines are of first order and the three phases are denoted lo (liquid ordered), so (solid ordered), and (diquid disorcered). The three phase ines meet in a tripie point. The horizontal line denotes a scan through the phase diagram at $J_{p}=0.491 \times 10^{-13} \mathrm{erg}$ with results as given in Fig. 3.

in the tenth conformational state. We identify this phase with the LE monolayer phase and we will refer to it as the liguid disordered (ld) phase in accordance with Refs. 27 and 33. The phase at high lateral pressures is characterised by Potts order and conformationally ordered chains, i.e., acyl chains in one of the gel-like conformational states. We identify this phase with the crystalline phase of the monolayer and we will refer to it as the solid ordered (so) phase. For $J_{p}<J \psi_{p}^{*}$ a second phase boundary appears and results in the presence of an intermediate phase which is characterized by conformationally ordered chains and Potts disorder. We identify this phase with the LC monolayer phase and we will refer to it as the liquid ordered (lo) phase. Figure 2 shows that in the case of coupled transitions, the so-ld phase line is slightly sensitive to the value of $J_{p}$.

A striking feature of the phase diagram in Fig. 1 is the very steep phase line separating the lo and so phases. The steepness of this line reflects the fact that the lateral pressure

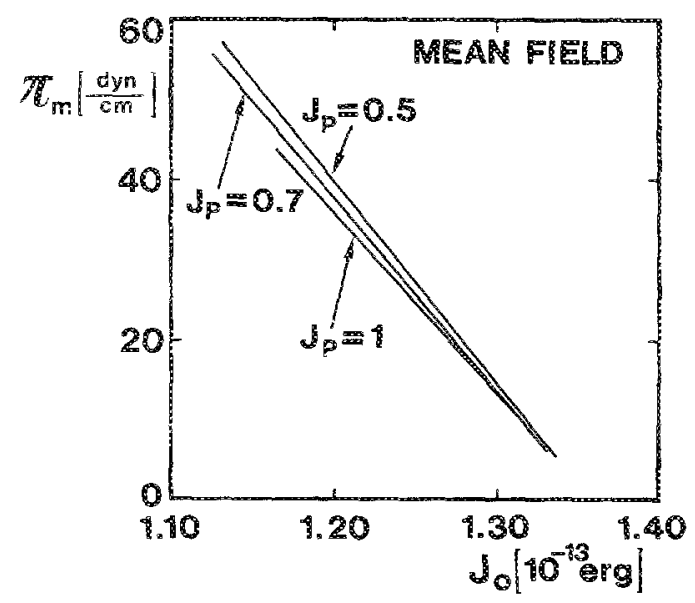

FIG. 2. Variation of mean-held main transition pressure $\Pi_{m}$ with van der Waals interaction constant $J_{0}$, for different values of the Potts coupling constant in the case of coupled transitions. 
becomes a weak thermodynamic variable once the acyl chains have undergone their conformational ordering transition. The physical reason for this, in terms of our model, is that $\mathrm{I}$ acts on the Potts variables only via the fluid conformational state. The position of the triple point moves up in pressure and towards higher values of $J_{p}$ as the temperature is raised. The consequence of this is that if the system is close to the rriple point, slight changes in temperature can switch the coupling of the two transitions on and of leading to dramatic changes in the phase behavior.

The effects of coupling and decoupling strongly manifest themseives on the isotherms. A series of mean-field isotherms in the case of $J_{P}=0.491 \times 10^{-13} \mathrm{erg}$ is given in Fig. 3 for several temperatures. For $T>25^{\circ} \mathrm{C}$, the two transitions are decoupled, cf. Fig. 1, and the two separate transitions are signalled by horizontal portions of the isotherm. Only at $30^{\circ} \mathrm{C}$ is the high-II transition seen on the scale of Fig. 3. For $T<25^{\circ} \mathrm{C}$, the two transitions are coupled. An approximate linear variation of the main transition pressure ${ }_{n} m$ with temperature is observed in the region of coupled transitions.

The mean-field results presented in this section were obtained for acyl-chain lengths of 16 carbon atoms. On the basis of knowledge of the variation of the parameters of the conformational Hamilonian ${ }^{19}$ we expect that the splitting between the two transitions will increase when the chain length is increased.

\section{N. COMPUTEA SMULATORS}

\section{A. Numertcal techniques}

The computer simulations are carried out by means of a Metropolis Monte Cario method ${ }^{34}$ for the constant- $T$ constan-1I ensemble. The excitation scheme follows that of single-site Glauber dynamics ${ }^{34}$ for which each trial move corresponds to a random assignnent of a conformational state and simultaneousiy a Potts state if the conformational state chosen is among the nine gel-like states. The simulations are performed on finite lattices of different sizes subject to toroidal periodic boundary conditions. The mator part of the results presented below is obtained for a system with $N=100 \times 100$ chains (or 5000 lipid molecules). Systematic finite-size studies show that the $100 \times 100$ system represents the thermodynamic limit as far as the properties corsidered

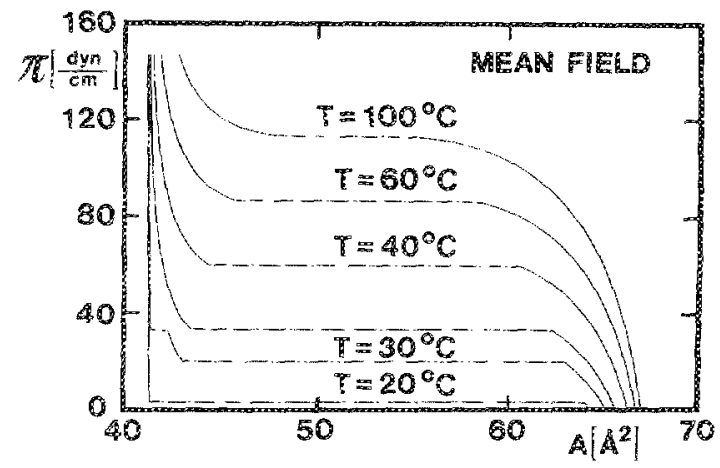

FIG. 3. Mean-field isotherms at $J_{p}=0.491 \times 10^{-1.3} \mathrm{erg}$, cf. Fig. 1, for a series of temperatures corresponding to cowpled as well as decoupled transitions. in this paper are concerned. The calculations are carried out for series of both increasing and decreasing lateral presseres in order to examine hysteresis effects. The number of Monte Carlo steps (MCS/S) generated per site is a measure of the statistics and is furthermore, via a master-equation interpretation, ${ }^{34}$ taken to be a time parameter in terms of which nonequilibrium effects and the dynamics of ordering processes may be studied. ${ }^{35}$

We have calculated the total internal energy and the average cross-sectional area per molecule. We have furthermore derived the isothermai specific heat and the area compressibility by means of the fluctuation-dissipation theorem. Phase transitions and phase boundaries are located by observing anomalies in the response functions. Due to the extensive demands in computer time for simulating the present model, we have restricted the numerical simulations to a single temperature, $T=30^{\circ} \mathrm{C}$, with a set of model parameters pertinent to DPPC. The value of $J_{0}$ is taken to be the same as that used for the mean-field calculations in See. $\mathrm{IHI} \mathrm{B}$.

\section{Phase diagram}

The computer-simulation resuits for the $\left(\Pi 1-J_{p}\right)$ phase diagram for $J_{0}=1.28 \times 10^{-13} \mathrm{erg}$ and $T=30^{\circ} \mathrm{C}$ are shown in F:g. 4. All phase boundaries correspond to first order transitions. It is seen that the Monte Carlo phase diagram has the same topology as the mean-field plase diagram in Fig. 1 with a triple point below which the lo phase becomes stable. The lo-so and som -ld phase lines are associated with pronounced hysteresis effects and they have been determined by using mainly small lattices in order to observe the relaxation out of the metastable states within a reasonable time. In particular, the steep boundary between the lo and so phases is difficult to determine accurately in a constant- $J_{p}$ calculation. Direct comparison between Figs. 1 and 4 shows that the neglect of fluctuations in the mean-field calculation leads to a suppression of the phase boundaries in terms of both $\Pi$ and $J_{p}$, as expected.

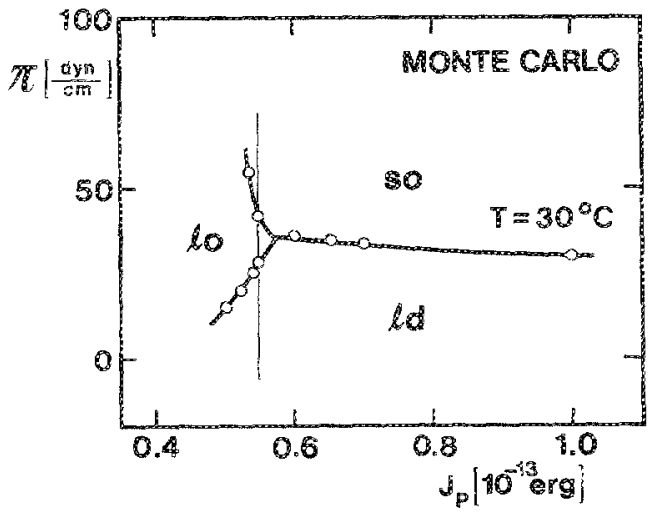

FIG. 4. Monte Carlo phase diagram, viz. Iateral pressure II vs Potts coupling constant $J_{P}$, at $T=-30^{\circ} \mathrm{C}$. All phase lines are of first order and the three phases are denoted to (liquid ordered), so (solid ordered), and Id (hiquid disordered). The three phase lines meer in a triple point. The horizontal line denotes a scan through the phase diagram at $J_{p}=0.55 \times 10^{-13}$ erg with results as given in Figs. 5-8. 


\section{Isotherms}

We have studied in detail the ordering phenomena along a single isotherm at $T=30^{\circ} \mathrm{C}$ in the case of decoupled transitions, $J_{p}=0.55 \times 10^{\cdots 13} \mathrm{erg}$, i.e., below the triple point in Fig. 4. At present, there seems no direct way of relating the value of $J_{p}$ to molecular properties of a given lipid monolayer. $J_{P}$ is therefore here considered to be an unknown model parameter. The thermaly driven ordering processes in the case of coupled transitions have previously been extensively studied in the context of ipid bilayer models. ${ }^{6,36}$ These previous studies revealed the interesting phenomenon of noncquilibrium interfacial melting when the system was prepared in a metastable polycrystalline aggregate and subsequently heated through the transition.

In Fig. 5 is shown the computer simulation data for the $30^{\circ} \mathrm{C}$ isotherm. The main branch of the isotherm (solid line) is obtained from a series of increasing lateral pressures staring from the ld phase. Each point is equilibrated $4000 \mathrm{MCS} /$ $\mathbf{S}$. For this equilibration time the isotherm is almost reversible in the la and ld phases with oniy a shigh hysteresis around the idolo transition. The relaxation in the transition region is very slow causing the isotherm to be nonhorizontal. ${ }^{24}$ In the so phase, however, the ordering kinetics is extremely slow and the equilibrium state camnot be attained for the $100 \times 100$ system within the maximum observation time ( $r \leqslant 8000 \mathrm{MCS} / \mathrm{S}$ ). For a smaller system, $N=30 \times 30$, it is possible to make the system go into the stable uniformly ordered Potts phase at high pressures. This is illustrated by the dotted line on Fig. 5. The calculations show that the loso transition is associated with a discontinuity in molecular area of approximately $\Delta A_{k \text {-so }} \simeq 0.8 \AA^{2}$. The remaining branch on Fig. 5 (the dasked line) corresponds to an isothermal scan performed by starting from a high-pressure uni-

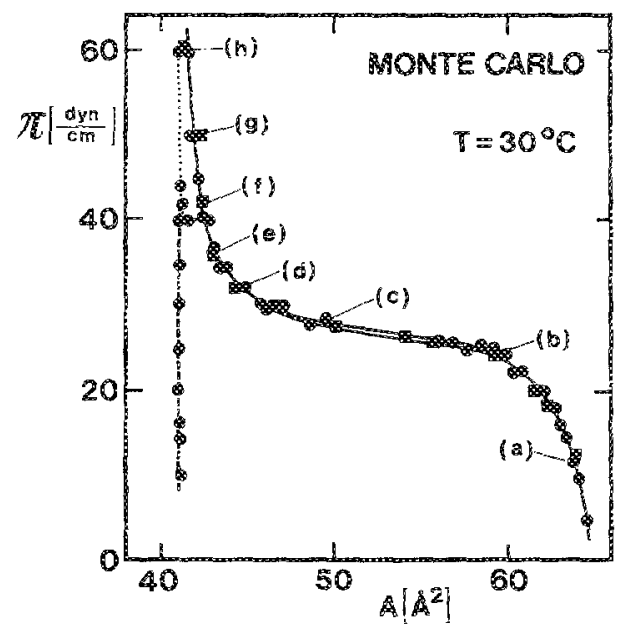

FIG. 5. Monte Carlo isotherm, viz. lateral pressure II vs cross-sectional arca per molecule, in the case of decoupled transitions, cf. Fig. 4. The results are obtained from Monte Carlo calculations on systems with 5000 (8) and 450 ( lipid molecules, respectively. The solid line denotes an isotherm which thigh pressures corresponds to a nonequilibrium monolayer with a domain structure. The dotted line refers to an equilibrium situation also at high pressures. The dashed line denotes at low pressures a metastable monolayer with a single uniformly Potts ordered crystallne domain. Points (a) (h) on the isotherm refer to the microconfigurations in Fig. 6. formy ordered Potts phase. It turns out to be impossible to make the system enter the Potts disordered phase lo for any positive lateral pressure, even for the smallest system studied, $N=30 \times 30$. Hence, the entire low-II part of this branch corresponcs to a metastable situation. The lateral pressure is simply a themodynamic variable which is too weak to afect the ordering dynamics appreciably within a reasonable time. This is in fact a general statement which also applies to metastable glassy phases of for example Lennard-Jones matter which is very difficult to anneal by hydrostatic pressure alone. ${ }^{37}$

From these observations we conclude that the high- $\Pi$ shoulder of the monolayer isotherm is strongly dependent on nonequilibrium effects. The longer the system is allowed to equilibriate in this region of the phase diagram, the less rounded the high-I shoulder will be. As pointed out in Sec. $\mathbb{V} \mathrm{D}$, the time evolution of this rounding may be interpreted in terms of a grain-growth process.

\section{Microscopic patern formation: Nonequilibium crystallazion and grain growth}

The microscopic phenomena underlying the various ordering processes as the monolayer is taken along the isotherm are ilustrated in Fig. 6. Snapshots of direct representations of microconfigurations characteristic of selected lateral pressures are shown in this figure. For pressures below the lo-so transition, these snapshots are typical of equilibrium. In contrast, the snapshots at higher pressures characterize nonequilibrium configurations for a system with a lateral-pressure history corresponding to $4000 \mathrm{MCS} / \mathrm{S}$ at each successive pressure of an increasing series as given in Fig. 5. For increasing $\Pi$, the microconfigurations refect the dramatic compression at the ld-lo transition where the acyl chains undergo the conformational ordering transition into the Potts disordered solid state lo. Upon further increase in pressure the system contracts only very shightly. The approach to the so phase is signalled by the appearance of clusters of chains in the same Pots state. These clusters are akin to density fuctuations. The cluster dynamics is very slow as the pressure changes.

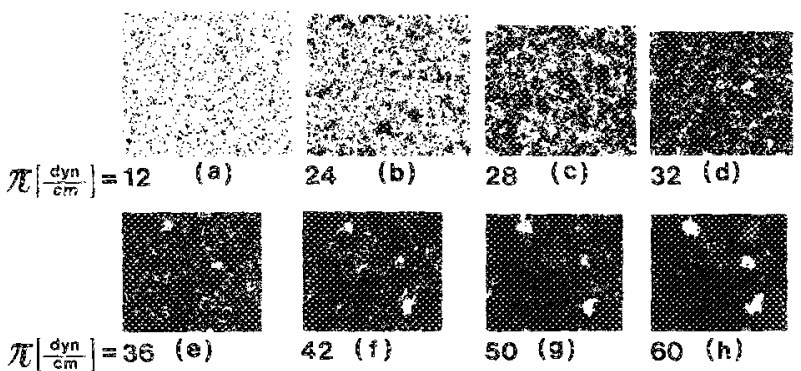

FIG. 6. Snapshots of microconfigurations for the points $(a)-(h)$ on the isotherm of Fig. 5. The configurations are obtained from Monte Carlo simulations on a lattice with $100 \times 100$ acyl chains. White areas indicate fuid domains and symbols indicate acyl chains in gel-like conformations, with each symbol labeling a crystal-orientational state (Potts state) of the acyl chain. The lattice parameter has been scaled so as to display the lateral compression of the monolayer as the pressure is increased from (a) to (h). Each configuration is typical for a relaxation time of $i=4000 \mathrm{MCS} / \mathrm{s}$. 


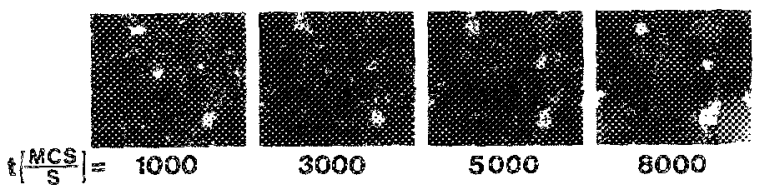

FIG. 7. Snapshots of the time evolution of microconfigurations on the isotherm of Fig. 5 for $I I=50 \mathrm{dyn} / \mathrm{cm}$. The notation is as in Fig. 6 .

When the lo-so phase line is crossed, these clusters grow persistently and eventually at long times or high pressures form the pattern of a polycrystalline aggregate characterized by a random network of grain boundaries separating competing domains with different Potts states. Due to the high degeneracy of the 30-state Potts model, the grains are compact. ${ }^{31}$ The domain pattern anneals extremely slowly in time, cf. Fig. 7. This is due to the fact that the iateral pressure only couples to the Potts ordering via the fluid states which have almost disappeared in the highly condensed lo and so phases. As seen in Fig. 7, there are even domains of the lo phase which are intercalated between Potts ordered grains. Moreover, the boundaries between the various ordered grains are typically widened (softened) by an interfacial region of Potts disorder. This disordered interfacial network screens the interaction between the ordered domains, lowers the driving force for grain growth, and consequently slows down the growth kinetics. Hence we have not found it possible within the available time span to study the late-time growth regime which we expect to be governed by the Lifshitz-Allen-Cahn growth law $^{35}$ for curvature-driven growth and nonconserved order parameter. The situation is quite different for quenches in temperature where the latetime asymptotic growth law can easily be probed. ${ }^{38}$

\section{E. Isothermal area compressibility}

The isothermal area compressibility $\propto(A)$ is derived from the fuctuation-dissipation theorem

$\kappa(A)=-\frac{1}{A} \frac{\partial A}{\partial \Pi}=\left(k_{B} T A\right)^{-1}\left(\left\langle A^{2}\right\rangle-\langle A\rangle^{2}\right)$.

The simulation results for $K(A)$ vs reciprocal area per molecule ( $\sim$ area density) are given in Fig. 8 which corresponds to the $30^{\circ} \mathrm{C}$ isotherm in Fig. 5. The compressibility is given for the main branch of the isotherm (the solid line), of. Fig. 5 , as well as for the branch which corresponds to data obtained by intiating the system in a uniformly ordered Potts phase characteristic of the so phase.

The area compressibility displayed in Fig. 8 has two distinct features which will prove of interest in connection with experimental observations. First, the lo-so transition is signaled by a shoulder in $\kappa(A)$. Second, the compressibility of the uniformly ordered Potts phase is considerably lower than that of the softer metastable grain structure, of. Fig. 7.

\section{COMPARISON WITH EXPERIMENTS ON PURE LIPID MONOLAYERS}

Before comparing the results of the present theoretical model study with experimental investigations of lipid mono-

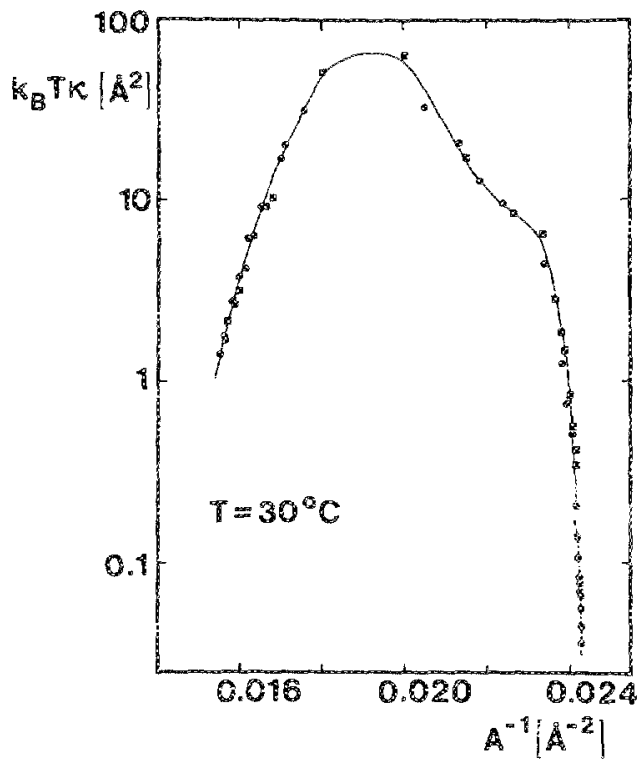

FG. 8. Isothernal area compressibility $\alpha(A)$ v inverse cross-sectional area per Higid molecule ( - area density) along the isotherm of Fig. 5 . The data are oftaned from Monte Carlo calculations on systens with 5000 (些) and 453 ( ) hipid molecules. The solid and dashed lines refar to the corresponeting branches of the isotherm in Fig. 5 .

layers we wish to emphasize that any such comparison should not be taken beyond a qualitative ievel. First, whereas we expect the model to provide a reliable representation of the acyl-chain conformational degrees of freedom, the fact that it is a lattice model excludes a fathulul representation of transiational degrees of freedon. Hence, the model is unable to describe two-dimensional hexatic phases with only longrange bond-orientational order. ${ }^{28}$ However, since the Potts variables, properly interpreted as crystal-orientation variables, may account for some of the topological consequences of crystallization, we suggest that the model put forward in this paper is useful for examining experimental observations within a framework of coupled and decoupled order parameters for the two sets of degrees of freedon. This is in particular true regarding the nonequilibrium properties which we believe to be of major importance for an understanding of lipid monolayer phase behavior. Second, since we are mainly interested in the general consequences of cecoupling of order parameters, we have made no attempt to fit the various model parameters to a specific lipid system. For convenience we have used a set of parameters which, as far as the conformational statistics of the lipid chains and the van der Wals interactions are concerned, will be pertinent to acyl chains with 16 monomers. In this respect, we model lipid monolayers of DPPC neglecting special effects such as chain tilting and details of molecular packing and defects. We expect, however, that the general tirdings of our study are valid for a larger class of Iipid monolayers.

A comparison with experimental results for lipid monolayers will be made on the following points: (i) general phase behavior, (ii) nature of the phase transition, (iii) shape of isotherms, and (iv) isothermal area compressibility.

(i) The experimental phase behavior of pure lipid mon- 
olayers is a highly controversial issue. ${ }^{39}$ In particular, the nature of the solid phases has been intensively discussed and several different suggestions have been made. $3.7-15$ The best understood system at the moment is probably the dimyristoyl phosphatidic acid (DMPA) monolayer which is the only monolayer for which in-plane structural information exists for the in sizu state. ${ }^{7,8}$ By combining the information obtained from synchrotron-X-ray scattering ${ }^{3,8}$ with that from fiuorescence microscopy, 8,9 the following picture emerges: Upon compression of the monolayer through the main transition $a t I_{m}$, solid domains are formed with a correlation length of the order of $10^{5} \AA$. These domains are not crystalline but have long-range bond-orientational order. ${ }^{8,9}$ Hence, it has been suggested ${ }^{8,39}$ that this phase may be a candidate for a hexatic phase peculiar to two-dimensional systems. As the pressure is further increased above II $c$, small crystalline regions are found to form with a coherence length of no more than $10^{2} \AA$. $^{78}$ The experimental findings for DMPA monolayers of an intermediate noncrystalline highly condensed phase are consistent with our theoretical predictions provided $y_{p}$ is below its value at the triple point, of. Fig. $i$ and the two transitions therefore are decoupled. Monolayers of polymerizable lipids could be candidates for systems with coupled transitions. ${ }^{7}$

(ii) It has been proposed that the transition at $I \mathrm{C}$ is continuous or of weakly first order. ${ }^{3,39}$ Our model study predicts a first-order transition at $\Pi_{c}$ but at the same time the computer-simulation results suggest that in the case of decoupled transitions, the ordering kinetics for the crystalline phase is extremely slow and may lead to a continuous smearing of the first-order transition, of. Fig. 5. This is a possible interpretation of the experimental results. Furthermore, the typical grain sizes seen above $\mathbb{B}_{c}$ in the computer simulations have linear dimensions comparable to those inferred from the synchrotron $x-r a y$ experiments. Furtherrore, the change in area at $I_{c}$ obtrained from the equilibrim simulations, $\Delta A_{\text {o-so }}$, is very similar to that derived from the synchrotron $x$-ray data for the change in the molecular nearestneighbor distance parameter. ${ }^{8}$ The theoretical findings for decoupled transitions of an extremely steep phase line for the lo-so transition, of. Figs. 1 and 4 , lead to the conclusion that II is a weak thermodynamic variable in this regime. We therefore sumise that only for lipid monolayers with special values of material constants, such as $J_{p}$, is it possible to find a thermodynamic stable crystalline phase at realistic lateral pressures. Furthermore, the region of stability of such a phase will be extremely sensitive to temperature, as suggested by the theoretical data of Fig. 1 .

(iii) Due to the very siow grain growth of the so phase both in the case of coupled and decoupled transitions, nonequilibrium effects will have a dramatic effect on the shape of the isotherms. Not oniy will long-lived themal density fuctuations, which are kinetically stabilized near $\mathbb{I}_{m}$, produce nonhorizontal isotherms at the chain-ordering transitions, ${ }^{24}$ but also the slow grain-growth kinetics near the lo-so phase boundary, cf Fig. 7, will broaden the high-II shoulder of the isothern. This is invariably observed experimentally, even in the most careful monolayer studies on ultrahigh purity lipids involving very precise pressure measurements and a high stanciard of humidity control. ${ }^{26}$ In these experiments, the isotherm at $\Pi_{m}$ is fully horizontal, but the broad high-I\} shoulder remains.

(iv) By comparing the macroscopic area compressibility obtained from the isotherms of DMPA and the corresponding microscopic compressibility obtained from the pressure variation of the crystal lattice parameter obtained from the synchrotron $x$-ray studies the striking results were found ${ }^{8}$ that the macroscopic compressibility is $2-3$ times larger than the microscopic compressibility. Heim er al. concluded that this difference is due to a possible infuence on the macroscopic compressibility of defect annealing, merging, and deformation of solid domains. This conchision is in excellent accordance with our computer simulation results, of. Fig. 6. Moreover, it is consistent with the numerical data for the compressibility in Fig. 8 if we take the compressibility of the branch corresponding to the homogeneously ordered Potts phase as that of microscopic crystalline domains and take the metastable branch corresponding to the soft polydomain Fotts state with glassy domain walls (cf. Fig. 7) as that of macroscopic polycrystalline aggregates. Two comments should be made regarding a more detailed comparison between Fig. 8 and the corresponding experimental compressibility taking DPPC as an example. ${ }^{3}$ First, the low-density compressibility is unrealistically small since the present model is not cesigned to describe the low-density phases of lipid monolayers. Second, the high-density compressibility of the model may also be too small due to the neglect of lipid-molecule tilt-degrees of freedom and defects in the head-group region. ${ }^{39}$ Despite these shortcomings of our model, the overall shape of $\kappa(A)$ vs $A^{-1}$, Fig. 8 , is very simiar to the experimental data.

\section{EFFECTS OF CHOLESTEROL}

\section{A. Cholesterol as a crystal breaker}

The advantage of the model for pure lipid monolayers described in Sec. II of this paper is that it gives a description of hipia monolayers based on two sets of two degrees of freedom, i.e, one for the chain conformations in terms of rotational isomerism and the other for the positional order in terms of the Potts variables. This description is particularly advantageous for mixed monolayers containing intrinsic impurities since a suitably extended model allows the impurity to afect both degrees of freedom differenty. Cholesterol, for example, can be regarded as a crystal breaker from the point of view of the positional degrees of freedom and a rigidifer of the acyl chains relative to the conformational degrees of freedom. A model along these lines has previously been proposed to describe the phase behavior of lipid bilayers containing intrinsic cholesterol molecules. ${ }^{33}$ In that model the crystal-breaking properties of cholesterol are accounted for by decoupling the Potts interaction between nearest-neighbor sites whenever a cholesterol molecule is involved. The hydrophobic interaction between cholesterol and another molecule is modelled in the spiric of lipic chain-chain interactions through a shape factor $\bar{l}_{c}$ which is a constant corresponding to the stiff cholesterol molecule. This requires the addition of the following two terms into the Hamitonian of Sec. II: 
$\mathscr{H}_{c c}=-\frac{\mathscr{L}_{0}}{2} \sum_{\langle i, j} \sum_{m, n=1}^{10} I_{c c} \mathscr{L}_{i c} \mathscr{L}_{j c}$

$\mathscr{H}_{c \mathrm{C}}=-\frac{J_{0}}{2} \sum_{(i, j)} \sum_{m, n=1}^{10} I_{c} I_{m}\left(\mathscr{L}_{i c} \mathscr{L}_{j m}+\mathscr{L}_{i n} \mathscr{L}_{j \mathrm{c}}\right)$.

Here $\mathscr{H}_{c c}$ is the Hamilonian representing the interaction between two neighboring cholesterol molecules and $I_{c c}$ is the strength of this interaction relative to $J_{0}$. $\mathscr{K}_{c c}$ is the interaction between a lipid acyl chain and a neighboring cholesterol molecule. In this work we assume that $I_{c c}=I_{c}^{2} . \mathscr{L}_{i c}$ is the site occupation variable for a cholesterol molecule.

The mean-field equations of Sec. II A can easily be adjusted to include a concentration of cholesterol molecules in the monolayer. First, the average value of $\mathscr{L}$ ic is related to the molar fraction of cholesterol, $x=\left\langle\mathscr{L}_{i c}\right\rangle /\left(2-\left\langle\mathscr{L}_{i c}\right\rangle\right)$. Next, the free energy of $\mathrm{Eq} .(6)$ is now a function of both $x$ and $T$ and the total entropy must include a mixing entropy term. Phase diagrams for lipid monolayers containing cholesterol can now be constructed using standard mathematical technigues. ${ }^{33}$

\section{Experimental results for lipidocholesterd monolays}

Lipid-cholesterol monolayers spread on air-water interfaces have been the subject of several experimental studies. Lateral pressure-surface area isotherms for these systems were obtained by Muller-Landau and Cadenhead ${ }^{40}$ and Abrecht et al. ${ }^{41}$ In addition, Aibrecht et al. constructed a tentative phase diagram for DPPC-cholesterol monolayers containing several possible coexistence regions. One of these regions gave an indication of phase separation between two hiquid phases, an almost pure lipid phase and a cholesterol rich phase. Subramaniam and McConneliz verified the occurrence of Iquid-liquid phase separation in their study of critical mixing of dimiristoyl phosphatidylcholine monolayers using fuorescence microscopy. Furthermore, they

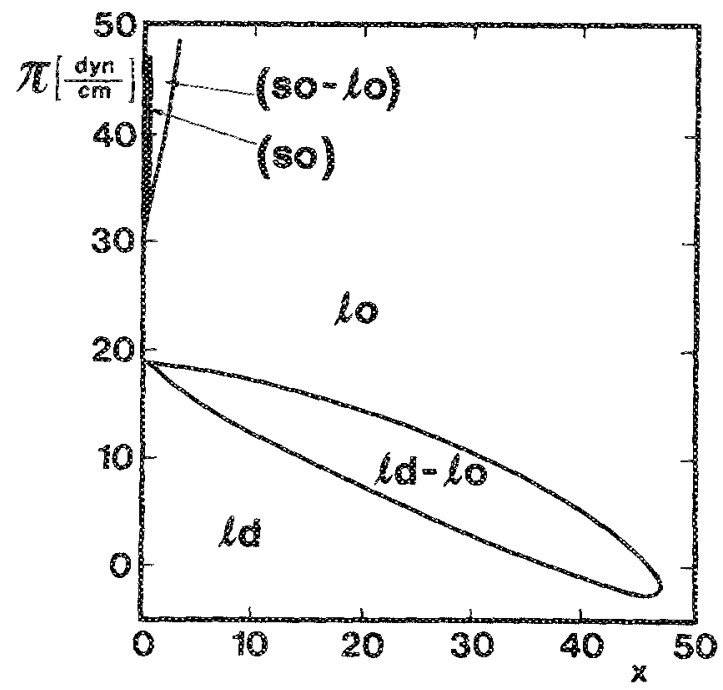

FlG. 9. Mean-field phase diagram, transition pressure vs cholesterol concentration $x$, for lipid-cholesterol monolayers in the case of decoupled transitions at $x=0$. showed that the two-phase coexistence disappears at a lateral pressure of about $10 \mathrm{dyn} / \mathrm{cm}$ for concentrations of $10-30$ mol \% cholesterol. Above this pressure the monolayers remain optically homogeneous for lateral pressures of up to 40 dyn/cm. Epiffuorescence studies ${ }^{42,43}$ of lipid-cholestero monolayers were used to investigate the LC/LE coexistence region at low cholesterol concentrations. These studies showed that the LC phase grows in the form of spiral domains in the coexistence region implying that the cholesterol molecules lie in the interface between the $L C$ and $L E$ phases and are responsible for the curvature.

\section{Theoretical phase diagram for lipid-chotesterol monoleyers}

The phase diagram appropriate for lipid-cholesterol monolayers is calculated in the mean-field approximation in the case of coupled as well as uncoupled transitions at zero cholesterol concentration. In the uncoupled case, the parameters chosen are $J_{0}=1.28 \times 10^{-13}$ ergs and $y_{p}=0.491 \times 10^{-13}$ ergs. As can be seen in Fig. 3, these values lead to a transition from the Id to the lo phase at $I=18$ dyn/cm and a transition from the lo to the so phase at about $I=35 \mathrm{dyn} / \mathrm{cm}$. Figure 9 gives the mean-field phase diagram for lipid-cholesterol monoiayers which is calculated using the above parameters. The phase transition at low lateral pressure broadens into a phase coexistence loop signaling a phase separation between the ld and lo phases. Part of the phase diagram found by Albrech et al. ${ }^{41}$ for DPPC. cholesterol monolayers can be interpreted in terms of the upper portion of the coexistence loop and the experimental results of Subramaniam and McConnell' imply the existence of the lower critical point of the phase coexistence region. The lateral pressure at which the upper transition occurs increases dramatically with increasing cholesterol concentration with only a very narrow stable region for the so phase. The upper transition takes piace at a very high lateral pressure which is a consequence of the weak dependence of the boundary between the 10 and so on lateral pressure in the phase diagram of Fig. 1 for the pure lipid monolayer. The experimental results of Albrecht et $a l^{41}$ exhibit a

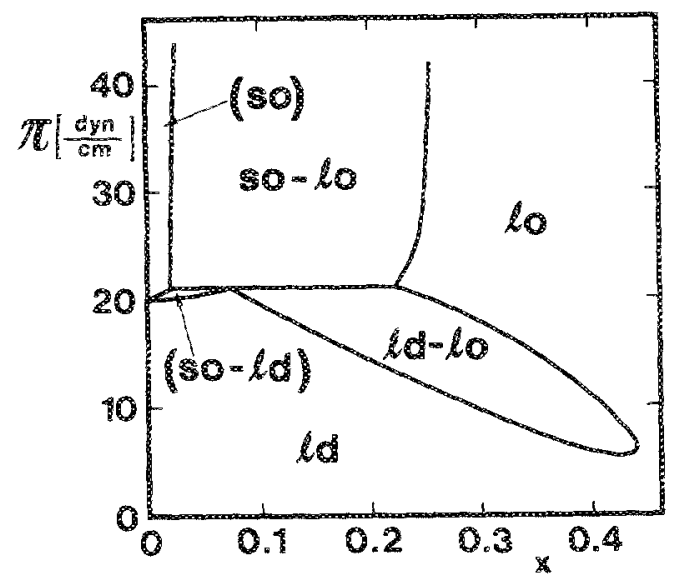

TO. 10. Mean-fieid phase diagram, transition pressure vs cholesterol concentration $x$, for lipid-cholesteroi monolayers in the case of coupled transitions at $x=0$. 
high-pressure phase boundary for DPPC-cholesterol monolayers but the behavior of this phase boundary is consider ably different from our results.

Figure 10 gives a mean-field phase diagram for lipidcholesterol monolayers in the case where there is only one phase transition in the pure monolayer from the so to the ld phase. The values of the parameters were the same as those used for the calculation of the phase diagram appropriate for lipid-cholesterol bilayers ${ }^{33}$ with the exception of the internal lateral pressure $\left(J_{0}=1.28 \times 10^{-13} \mathrm{erg}\right.$ and $J_{P}=0.525 \times 10^{-13} \mathrm{erg}$ ). The resultant monolayer phase diagram is essentially the inverse of the bilayer phase diagram $^{33}$ in terms of its shape and it is completely different from the phase diagram shown in Fig. 9. While there is at present no experimental evidence for such a phase diagram, it is not inconceivable that there are pure lipid monolayers exhibiting only one so-ld phase transition. As previously pointed out, possible candidates for a single so-ld transition are monolayers of polymerizable lipids. ${ }^{7}$

Clearly our model in its present form can shed no light on the formation of spiral domains ${ }^{42.43}$ However, it clarifies certain aspects of the experimental phase diagrams of lipidcholesterol monolayers and it predicts the occurrence of a liquid-liquid phase separation as found by Subramaniam and McConnell. ${ }^{42}$ A more complete experimental phase dirgram for lipid-cholesterol monolayers is required before a more detailed theoretical analysis is warranted and a proper assessment can be made of the theoretical model proposed in the present paper.

\section{CONGLUSIONS}

In this paper we have presented a theoretical model study of the phase behavior of lipid monolayers spread on the air/water interface. The study focuses attention on consequences of the fact that lipid monolayers are a unique class of physical systems which possess two different mechanisms for ordering, one being the conformational ordering of the acyl lipid chains via rotational isomerism, and the other being crystallization of the monolayer involving the transliational degrees of freedom. The latter mechanism leads to formation of crystalline domains with a large number of different orientations. The main emphasis of the paper has been to determine the influence on the phase behavior of decoupling of the two orciering mechanisms, with particular emphasis on the nonequilibrimm behavior.

It is pointed out that decouping of conformational ordering and crystallization is experimentaily observed in certain lipid monolayers ${ }^{7,8}$ and it is suggested that the observed lack of long-range crystallinity at high pressures may be due to a particularly slow kinetics of the pressure-driven crystallization process. Similarly, an interpretation is given of the commonly observed nonhorizontal isotherms and rounded high-pressure shoulders of the isotherms as being due to a slow nonequilibrium grain-growth process.

Cholesterol is shown, in accordance with experiments, ${ }^{40-42}$ to possess a unique capacity for providing a molecular mechanism for decoupling acyl-chain ordering processes and crystallization leading to the peculiar phase behavior of lipid monolayers containing cholesterol.
The results of the present study also have relevance for the phase behavior of lipid bilayers ${ }^{6,36}$ which are believed to represent the case of coupied ordering mechanisms in the absence of cholesterol. In particular, it was recently demonstrated experimentally, $44,4,5$ and subsequently interpreted theoretically, ${ }^{33}$ that cholesterol in lipid bilayers acts as a crystal breaker which decouples the two ordering mechanisms. At high cholesterol concentrations this decoupling leads to a peculiar homogeneous phase which, from the point of view of bulk mechanical properties, is a liquid ${ }^{46}$ but which at the same time has a high degree of acyl-chain ordering and cohesive strength. It is likely that cholesterol as a decoupler in this respect is a unique molecule of nature and an indispensible prerequisite for imparting to biological membranes their peculiar physical properties. ${ }^{47}$

\section{ACKNOWLEDGMENTS}

This work was supported by the Danish Natural Science Research Council under Grants Nos. Inr. 11-5176 and 5.21.99.72, by NSERC of Canada, and by Le FCAC du Quebec. One of us (M.J.Z.) wishes to thank Vera and Carl Johan Michaesens Legat for funding a visiting professorship at The Technical University of Denmark. A stimulating discussion with Erich Sackmann is gratefully acknowledged.

'P. K. Adam, Proc. R. Soc. London Ser. A. 10R, 516 (1922)

${ }^{2} \mathrm{G}$. F. Gaines, Jr., Insoluble Monolayers at Liquid-Gas Interfaces (Wiley, New York, 1966).

30. Albrecht, H. Gruler, and E. Sackmann, F. Phys. (Paris) 39, 301 $(1978)$.

4R. C. MacDonald and S. A. Simon, Proc. Natl. Acad. Sci. USA 84, 4089 (1987).

${ }^{5} \mathrm{M}$. Cafirey, Biochemistry 24, 4826 (1985).

"M. J. Zuckemann and O. G. Mouritsen, Eur. Biophys. J. 15,77 (1987). ${ }^{7}$ N. Kjnet, J. Als-Nielsen, C. A. Heim, L. A. Laxtuber, and H. Möhwald, Phys. Rav. Eett. 58, 2224 (1987).

${ }^{8} \mathrm{C}$. A. Helm, H. Mohwald K. Kjaer, and J. Als-Nielsen, Biophys. I. 52 , 381 (1987).

${ }^{9}$ V. T. Moy, D. I. Keller, H. E. Gaub, and L. M. McConnell, J. Phys. Chem. 90,3198 (1986).

10. Fischer and E. Sackmann, J. Phys. (Paris) 45, 517 (1984).

${ }^{11}$ A. Fischer, M. Lösche, and E. Sackmann, J. Phys, Lett. (Paris) 45, L-785 (1984).

${ }^{12} \mathrm{~V}$. von Tscharner and H. M. McConnell, Bionhys. 3. 36, 409 (1981).

${ }^{13}$ M. Lösche and H. Möhwald, Coll. Surf. 10, 217 (1984).

1. IV. Lösche and H. Möhwald, Eur. Biophys. J, 11, 35 (1984).

15. M. Weis and H. M. McConnell, Nature 316, 47 (1984).

${ }^{36} \mathrm{C}$. A. Helm, H. Möhwald, K. Kjaer, and J. Als-Nielsen, Europhys. Let 4, 697 (1987).

${ }^{17}$ T. Rasing, Y. R. Shen, M. W. Kim, and \$. Grubb, Phys. Rev. Lett. 55, $2903(1985)$.

${ }^{18} \mathrm{P}$. Guyot-Siomest, I. H. Hunt, and Y. R. Shen, Phys. Rev. Lett. 59, 1597 (1987).

${ }^{19}$ A. Cailié, D. Pink, F. de Verteuil, and M. J. Zuckermann, Can. J. Phys. 58, 581 (1980).

${ }^{20}$ A. Georgallas and D. A. Pink, J. Coll. Int. Sci. 89, 107 (1982).

${ }^{21}$ A. Georgallas and D. A. Pink, Can. J. Phys.60, 1678 (1982).

22I. E. Nagle, Faraday Discuss. Chem. Soc. 81, 151 (1986).

${ }^{23}$ M. J. Zuckermann, D. A. Pink, M. Costas, and B. C. Sanctuary, J. Chem. Phys. 76,4206 (1982).

${ }^{24}$ O. G. Mouritsen, J. H. Ipsen, and M. J. Zuckermann, J. Col. Int. Sci. 129, 32 (1989).

${ }^{25}$ D. A. Pink, T. J. Green, and D. Chapman, 3 iochemistry 19, 349 (1980). 
${ }^{26}$ N. R. Pallas and B. A. Pethica, Langmuir 1, 509 (1985).

${ }^{27}$ O. G. Mouritsen and M. J. Zuckermann, Chem. Phys. Lett. 135,294 (1987).

${ }^{28}$ D. R. Nelson, Phys. Rev. B 26, 2902 (1983).

${ }^{219}$ S. H. Northrup and M. S. Curvin, J. Phys. Chem. 89, 4707 (1985).

${ }^{30}$ J. EF. Bareman, G. Cardini, and M. L. Klein, Phys. Rev. Lett. 68,2152 (1988).

"P. S. Sahni, G. S. Grest, and M. Y. Anderson, Phys. Rev. Lett. \$0, 263 (1983).

${ }^{32}$ O. G. Mouritsen and M. J. Zuckermann, Eur. Biophys. J. 12, 75 (1985).

33J. H. Ipsen, G. Karlström, O. G. Mouritsen, H. Wennerström, and M. J. Zuckermann, Biochim. Biophys. Acta $\$ 05,162$ (1987).

${ }^{34}$ O. G. Mouritsen, Computer Studies of Phase Transitions and Critical Phenomena (Springer, New York, 1984).

${ }^{35}$ J. D. Gunton, M. San Miguel, and P. S. Sahni, in Phase Tronsitions and Critical Phenomena, edited by C. Domb and J. L. Lebowitz, (Academic, Now York, 1984), Vol. VII, p. 267.

${ }^{36}$ O. G. Mosritsen and M. J. Zuckermann, Phys. Rev. Lett. 58,389 (1987).

${ }^{37}$ R. M. J. Cotterill and J. U. Madsen, Phys. Rev. B 33, 262 (1986).

${ }^{38} \mathrm{O}$. G. Mouritsen, in Annealing Processes-Recovery, Recrystallization and
Grain Growth, edited by N. Hansen, D. Juul Jensen, T. Leffers, and B. Raiph (Riss National Laboratory, Roskilde, 1986), p. 457.

${ }^{39}$ E. Sackmann, A. Fischer, and W. Frey, in Physics of A mphiphilic Layers, edited by J. Meunier, D. Langevin, and N. Boccara (Springer, New York, 1987), p. 25.

${ }^{40}$ F. Müher-Landau and D. A. Cadenhead, Chem. Phys. Lipidis 25, 299 (1979).

10. Albrecht, H. Gruler, and E. Sackmann, 5 . Coll. Int. Sci. 79, 319 (1981).

${ }^{42}$ S. Subramaniam and H. M. McConnell, J. Phys. Chem. 91, 1715 (1987).

${ }^{43}$ W. M. Keckl, M. Lösche, D. A. Cadenhead, and H. Möhwald, Eur. Biophys. J. 14, 11 (1986).

44J. H. Davis, in Proc. Int. School Phys. Enrico Fermi on Medical Applications of NMR, edited by B. Maraviglia (North-Holland, Ansterdam, 1988), p. 302.

${ }^{45}$ M. R. Vist, MSc. thesis, University of Guelph, Ontaric, Canada, 1984.

${ }^{46}$ D. Needhazi, T. J. MeIntosh, and E. Evans, Biochemistry 27, 4668 (1988).

47M. Bloom and O. G. Mouritsen, Can. J. Chem. 66, 706 (1988). 